Check for updates

Cite this: RSC Adv., 2018, 8, 4417

\title{
Spin-dependent and spin-independent channels of electrical transport in perovskite manganites
}

\author{
J. J. Qian, ${ }^{a}$ W. H. Qi, ${ }^{a}$ Z. Z. Li, ${ }^{a}$ L. Ma, ${ }^{a}$ G. D. Tang, (D) *ab Y. N. Du, ${ }^{a}$ M. Y. Chen, ${ }^{a}$ \\ G. H. Wu $u^{b}$ and F. X. Hu (DD
}

A model with two channels of electrical transport (TCET) for perovskite manganites is proposed, and it is described by an equivalent device with two current-carrier channels. In one channel, there is a spin-independent resistor $\left(R_{3}\right)$ with an equivalent resistivity of $\rho_{3}$. In the other channel, there are two spin-dependent resistors in series $\left(R_{1}\right.$ and $\left.R_{2}\right)$ with an equivalent resistivity of $\rho_{1}+\rho_{2}$. The component $\rho_{1}$ includes residual resistivity and the resistivity contributed by crystal-lattice scattering. The other component of the equivalent resistivity, $\rho_{2}$, originates from the spin orientations of the itinerant electrons and the local electrons of the outer $O 2 p$ and $\mathrm{Mn} \mathrm{3d}$ orbits that deviate from the orientation of their ground states when the test temperature is close to the Curie temperature. Using this model, we fitted the experimental curves of the resistivity versus test temperature for single-crystalline $\mathrm{La}_{1-x} \mathrm{Sr}_{x} \mathrm{MnO}_{3}(0.00 \leq x \leq 0.40)$ and polycrystalline $\mathrm{La}_{0.6} \mathrm{Sr}_{0.4} \mathrm{Mn}_{1-x} \mathrm{Fe}_{x} \mathrm{O}_{3}$ $(0.00 \leq x \leq 0.30)$. In addition, we investigated the effects of the fraction of the antiferromagnetic phase, scattering at the crystallite interfaces, and the crystal-cell constants on the samples' resistivity. The physical mechanism of the TCET model was explained using an $\mathrm{O} 2 \mathrm{p}$ itinerant-electron model, which has been used in other studies to explain the magnetic ordering of several series of spinel ferrites and perovskite manganites.

Received 29th November 2017 Accepted 17th January 2018

DOI: 10.1039/c7ra12878a

rsc.li/rsc-advances anions. When compared with an $\mathrm{O}^{2-}$ anion $\left(2 \mathrm{~s}^{2} 2 \mathrm{p}^{6}\right)$ with a closed outer electron shell, it is clear that a p hole exists in the outer orbit of an $\mathrm{O}^{1-}$ anion $\left(2 \mathrm{~s}^{2} 2 \mathrm{p}^{5}\right)$. This fact was further confirmed by theoretical calculations ${ }^{22,23}$ and experiments. ${ }^{24-26}$

Taking into account that there are both $\mathrm{O}^{1-}$ and $\mathrm{O}^{2-}$ anions in oxides, ${ }^{22-27}$ our group has proposed an $\mathrm{O} 2 \mathrm{p}$ itinerant-electron model for magnetic oxides (IEO model), ${ }^{28,29}$ which is similar to the $\mathrm{O} 2 \mathrm{p}$ hole model proposed by Alexandrov et al. ${ }^{13,14}$ Using the IEO model, our group studied the relationship between the magnetic moment and cation distribution of several series of $\mathrm{Cr}$ (Ti, Mn)-doped spinel ferrites. ${ }^{28-36}$ We also studied the magnetic ordering in perovskite manganites. ${ }^{37-40}$

In this study, we used a model of two channels of electrical transport (TCET), which was derived from the IEO model, to fit the temperature dependence of the resistivity for two series of perovskite manganites: single crystallines $\mathrm{La}_{1-x} \mathrm{Sr}_{x} \mathrm{MnO}_{3}(0.00$ $\leq x \leq 0.40$ ) reported by Urushibara et $a l .{ }^{41}$ and polycrystallines $\mathrm{La}_{0.6} \mathrm{Sr}_{0.4} \mathrm{Mn}_{1-x} \mathrm{Fe}_{x} \mathrm{O}_{3}(0.00 \leq x \leq 0.30)$ prepared in this work. In addition, we will discuss the effects of the proportion of the antiferromagnetic phase, scattering at crystallite interfaces, and crystal-cell constants on the samples' resistivity.

\section{Experiment and results}

The polycrystalline perovskites examined in this study, $\mathrm{La}_{0.60^{-}}$ $\mathrm{Sr}_{0.40} \mathrm{Fe}_{x} \mathrm{Mn}_{1-x} \mathrm{O}_{3}(0 \leq x \leq 0.3)$, were prepared using the sol-gel method: ${ }^{42,43}$ (i) stoichiometric amounts of $\mathrm{La}_{2} \mathrm{O}_{3}$ (purity: $99.99 \%$, fired in air at $1073 \mathrm{~K}$ for $3 \mathrm{~h}$ before it was used),
${ }^{a}$ Hebei Advanced Thin Film Laboratory, Department of Physics, Hebei Normal University, Shijiazhuang City 050024, People's Republic of China.E-mail: tanggd@ hebtu.edu.cn; Tel: +8631180787330

${ }^{b}$ State Key Laboratory of Magnetism, Institute of Physics, Chinese Academy of Sciences, Beijing 100190, People's Republic of China 

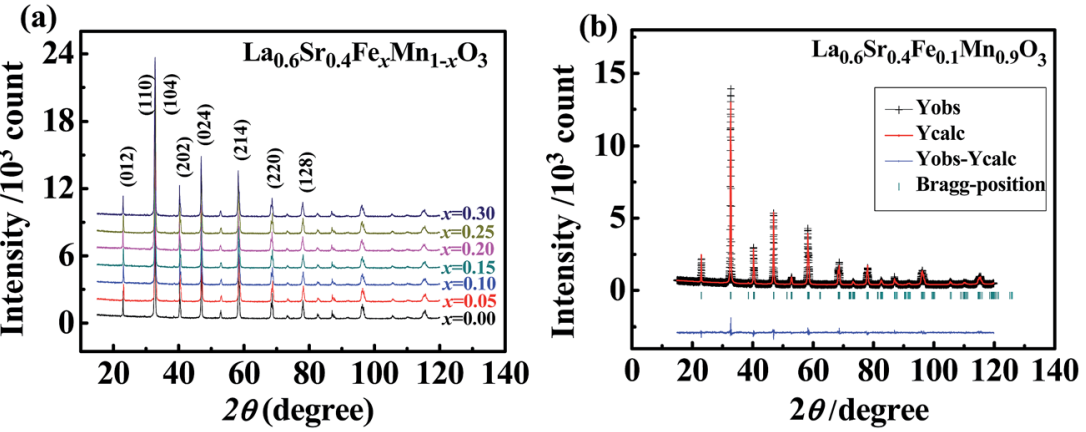

Fig. 1 (a) Powder XRD patterns of $\mathrm{La}_{0.6} \mathrm{Sr}_{0.4} \mathrm{Fe}_{X} \mathrm{Mn}_{1-x} \mathrm{O}_{3}$. (b) Fitted and observed XRD patterns for the sample $\mathrm{La}_{0.6} \mathrm{Sr}_{0.4} \mathrm{Fe}_{0.1} \mathrm{Mn}_{0.9} \mathrm{O}_{3}$, obtained using the Fullprof software. The crosses represent the observed data, and the curve represents the fitted results; the vertical bars represent the Bragg sites for the space group $R 3 c$. The difference between the observed and fitted patterns is shown at the bottom.

$\mathrm{Sr}\left(\mathrm{NO}_{3}\right)_{2}$ (purity: 99.5\%) powder, and a $\mathrm{Mn}\left(\mathrm{NO}_{3}\right)_{2}$ solution (purity: 50\%) were dissolved in a dilute $\mathrm{HNO}_{3}$ solution at $343 \mathrm{~K}$. Citric acid and ethylene glycol were added to the mixed solution as complexing agents until a completely homogeneous transparent solution was obtained. The molar ratio of citric acid (or ethylene glycol) to the perovskite molecule was $6: 1$. (ii) The mixture was then evaporated at $363 \mathrm{~K}$ for $24 \mathrm{~h}$ in a water bath until a highly viscous residue was formed. Upon further heating at $373 \mathrm{~K}$ for $12 \mathrm{~h}$ and then at $473 \mathrm{~K}$ for $24 \mathrm{~h}$, a gel developed. The gel was ground. (iii) All ground gel samples of different compositions were further heat treated. The temperature was increased from 473 to $573 \mathrm{~K}$, in increments of $25 \mathrm{~K}$, with $45 \mathrm{~min}$ spent at each step. Next, the temperature was increased from 573 to $673 \mathrm{~K}$ in increments of $20 \mathrm{~K}$, with 50 min spent at each step. Finally, the temperature was increased from 673 to $773 \mathrm{~K}$ in increments of $10 \mathrm{~K}$, with $1 \mathrm{~h}$ spent at each step. The samples were finely ground for $30 \mathrm{~min}$ to obtain a homogeneous mixture. After that, the samples were calcined at $873 \mathrm{~K}$ for $5 \mathrm{~h}$ to eliminate the organic material, ground, and calcined again at $1073 \mathrm{~K}$ for $10 \mathrm{~h}$ to eliminate any residual organic material. Each sample was then divided into two parts to measure their magnetic and electrical properties, and they were subjected to further heat treatment using slightly different methods.

To prepare the samples used to measure the magnetic properties, the powder obtained after grinding was pressed into pellets with a diameter of $13 \mathrm{~mm}$ and thickness of about $2 \mathrm{~mm}$ under a pressure of 11 ton per $\mathrm{cm}^{2}$. The pellets were calcined at
$1273 \mathrm{~K}$ for $10 \mathrm{~h}$, after which powder samples were obtained by grinding.

To prepare the samples used to measure the electricaltransport properties, the powder obtained after grinding was pressed into pellets under a pressure of 10 ton per $\mathrm{cm}^{2}$. The pellet samples were then sintered at $1573 \mathrm{~K}$ for $24 \mathrm{~h}$.

Structural characterization was carried out by analyzing $\mathrm{X}$ ray diffraction (XRD) patterns, which were measured using an $\mathrm{X}$-ray diffractometer (X'Pert Pro, PANalytical, The Netherlands) with $\mathrm{Cu} \mathrm{K} \mathrm{K}_{\alpha}$ radiation $(\lambda=1.5406 \AA)$. The data were collected in the $2 \theta$ range of $15^{\circ}$ to $120^{\circ}$ in increments of $0.0167^{\circ}$. The field and temperature dependence of the specific magnetization, $\sigma(H)$ and $\sigma(T)$, were measured using a physical-property measurement system (PPMS, Quantum Design, Inc., USA). Using electrical-transport characterization equipment (Versa Lab, Quantum Design, Inc., USA), the temperature dependence of the electrical resistivity $\rho(T)$ was measured by the conventional four-point probe technique at $50 \mathrm{~K}$.

Fig. 1(a) shows the XRD patterns, which indicate that all the samples had only a single $\mathrm{ABO}_{3}$ perovskite phase with the space group $R \overline{3} c$. The XRD patterns were fitted using the Fullprof software (Institut Laue-Langevin, France). The goodness-of-fit factor $(s \leq 1.37)$ is listed for all samples in Table 1. As an example, the fitted result for the $\mathrm{La}_{0.60} \mathrm{Sr}_{0.40^{-}}$ $\mathrm{Fe}_{0.1} \mathrm{Mn}_{0.9} \mathrm{O}_{3}$ sample is shown in Fig. $1(\mathrm{~b})$. The fitted result; the dependence on the Fe-doping level, $x$; the lattice parameters, $a$ and $c$; the crystal-cell volume, $v$; the $\mathrm{Mn}-\mathrm{O}$ bond

Table 1 Crystal structural parameters of $\mathrm{La}_{0.6} \mathrm{Sr}_{0.4} \mathrm{Fe}_{x} \mathrm{Mn}_{1-x} \mathrm{O}_{3}$ at room temperature: $a$ and $c$ are the cell constants, $v$ is the cell volume, $d_{\mathrm{B}-O}$ and $\Theta$ are the $\mathrm{Mn}-\mathrm{O}$ distance and $\mathrm{Mn}-\mathrm{O}-\mathrm{Mn}$ bond angle, all which were obtained by fitting the XRD patterns; $s$ is the goodness-of-fit factor. $\sigma_{\mathrm{S}}$ and $\mu_{\text {obs }}$ are the specific saturation magnetization and the average molecular magnetic moment at $10 \mathrm{~K}$, respectively. $T_{\mathrm{C}}$ and $T_{\mathrm{N}}$ are the Curie temperature and the Néel temperature, respectively; $T_{M I}$ is the temperature at which the resistivity has the maximum value

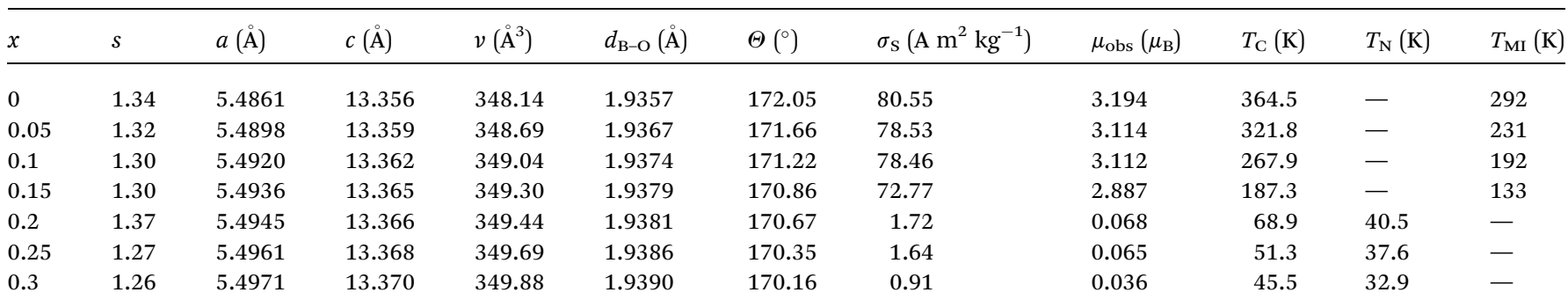



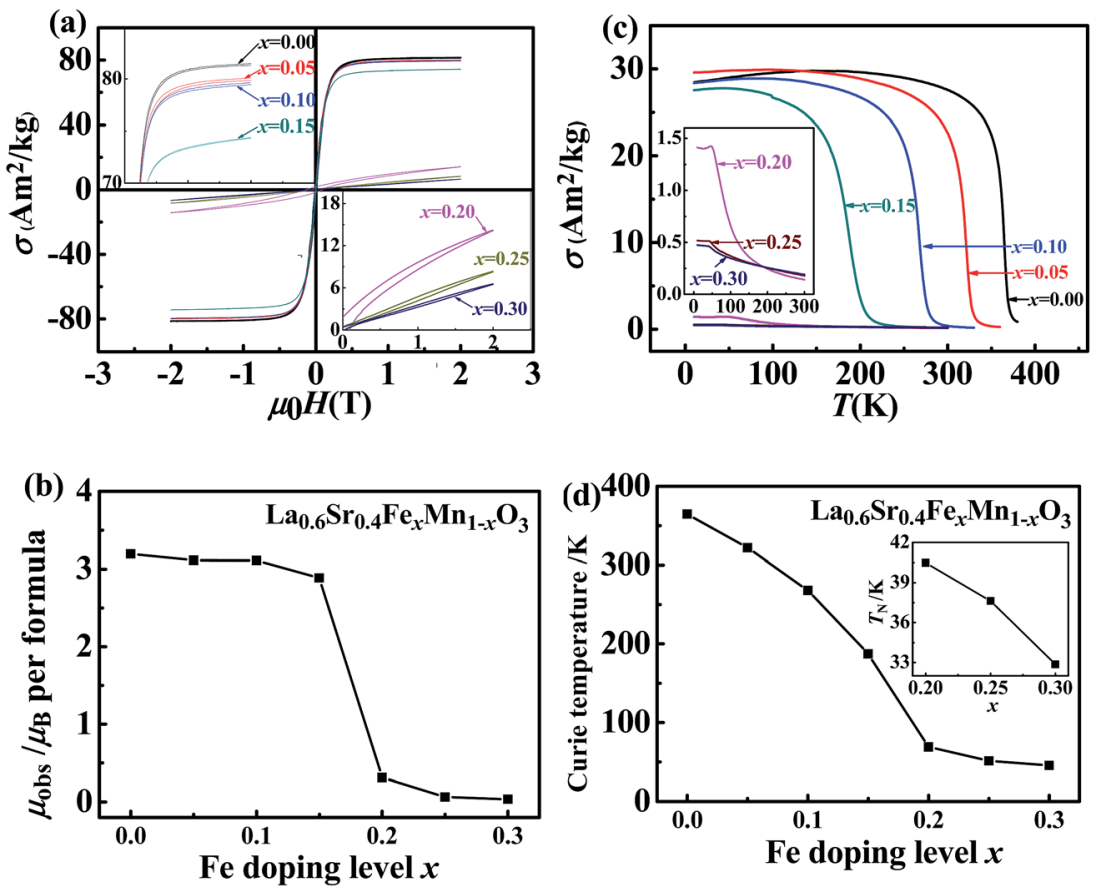

Fig. 2 Magnetic properties of $\mathrm{La}_{0.6} \mathrm{Sr}_{0.4} \mathrm{Fe}_{x} \mathrm{Mn}_{1-x} \mathrm{O}_{3}$ : (a) magnetic hysteresis loops at $10 \mathrm{~K}$; (b) magnetic moment, $\mu_{\mathrm{obs}}$, at $10 \mathrm{~K}$ as a function of $\mathrm{Fe}$ content, $x$; (c) temperature dependence of the specific magnetization, $\sigma$, at an applied magnetic field of $0.05 \mathrm{~T}$; (d) magnetic transition temperature as a function of Fe content, $x$. The insert of (c) enhanced the variations of the curves. The insert of (d) shows the Néel temperature, $T_{N}$, as a function of Fe content, $x$.

length, $d_{\mathrm{B}-\mathrm{O}}$; and the $\mathrm{Mn}-\mathrm{O}-\mathrm{Mn}$ bond angle, $\Theta$, are listed in Table 1. It can be seen that the values of these parameters slightly varied: the values of $a, c, v$, and $d_{\mathrm{B}-\mathrm{O}}$ increased with increasing $x$; and the value of $\Theta$ decreased with increasing $x$. The equivalent cubic cell constants $\left(a_{\mathrm{e}}\right)$ per formula, calculated using the crystal-cell volume, $v$, will be used to discuss the samples' resistivity in Section 4.4.

Fig. 2(a) shows the magnetic hysteresis loops of the samples measured at $10 \mathrm{~K}$. Using the specific saturation magnetization, $\sigma_{\mathrm{S}}$, at $10 \mathrm{~K}$, the average values of the molecular magnetic moment, $\mu_{\text {obs }}$, were obtained. Fig. 2(b) shows the curve of $\mu_{\text {obs }}$ versus $x$. Fig. 2(c) shows the curves of $\sigma$ versus $T$ under an applied magnetic field of $0.05 \mathrm{~T}$. The values of the Curie temperature, $T_{\mathrm{C}}$, defined as the temperature at which $\mathrm{d} \sigma / \mathrm{d} T$ reaches its minimum value, are shown in Fig. $2(\mathrm{~d})$. In

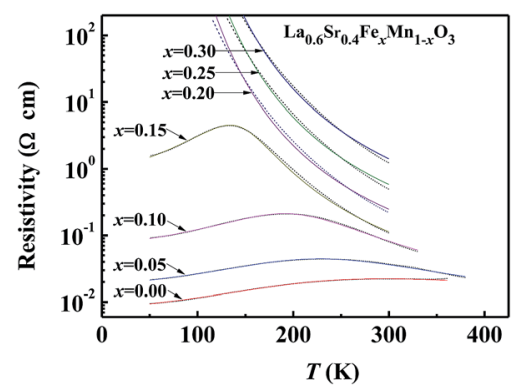

Fig. 3 Temperature dependence of electrical resistivity of the La $a_{0.6}$ $\mathrm{Sr}_{0.4} \mathrm{Fe}_{x} \mathrm{Mn}_{1-x} \mathrm{O}_{3}$ samples. The data points represent the observed values, and the curves represent the fitted results. addition, the inset in Fig. 2(c) shows that there was a different Néel temperature, $T_{\mathrm{N}}$, for each sample with $x \geq 0.20$. Below $T_{\mathrm{N}}$, the value of $\sigma$ exhibited no significant change; above $T_{\mathrm{N}}$, the value of $\sigma$ decreased distinctly. The variation of $T_{\mathrm{N}}$ with $x$ is shown in the inset in Fig. 2(d). The values of $\mu_{\mathrm{obs}}, T_{\mathrm{C}}$, and $T_{\mathrm{N}}$ are listed in Table 1.

The data points in Fig. 3 show the temperature dependence of the electrical resistivity, $\rho$, of the samples, and it can be seen that $\rho$ increased with increasing $x$. In addition, there was a resistivity peak at a characteristic temperature, $T_{\mathrm{MI}}$, for each sample when $x \leq 0.15$, and these characteristic temperatures are listed in Table 1. The curves in Fig. 3 also show the fitted results, which will be discussed in the following sections.

The following trends can be observed in Fig. 2 and 3: (i) when $x \leq 0.15$, the samples were ferromagnetic conductors. The value of $\mu_{\mathrm{obs}}$ decreased slowly from $3.194 \mu_{\mathrm{B}}(x=0.00)$ to $2.887 \mu_{\mathrm{B}}(x=0.15)$; the value of $T_{\mathrm{C}}$ decreased rapidly from $364.5 \mathrm{~K}(x=0.00)$ to $187.3 \mathrm{~K}(x=0.15)$; the maximum resistivity increased from $0.0222 \Omega \mathrm{cm}(x=0.00)$ to $4.47 \Omega \mathrm{cm}(x=$ 0.15 ). (ii) When $x \geq 0.20$, the samples were semiconductors with both an antiferromagnetic phase and a ferromagnetic phase. The resistivity increased rapidly with decreasing temperature, exceeding $100 \Omega \mathrm{cm}$ when the test temperature was below $123.6,140.2$, and $159.3 \mathrm{~K}$, when $x=0.20,0.25$, and 0.30 , respectively. In addition, when $x=0.20,0.25$, and 0.30 , respectively, the values of $T_{\mathrm{N}}$ of the antiferromagnetic phase were $40.5,37.6$, and $32.9 \mathrm{~K}$; the Curie temperatures of the ferromagnetic phase were $68.9,51.3$, and $45.5 \mathrm{~K}$; and the $\mu_{\mathrm{obs}}$ values were $0.068,0.065$, and $0.036 \mu_{\mathrm{B}}$. 


\section{Model with two channels of electrical-transport (TCET) for perovskite manganites}

In order to explain the electrical-transport properties of perovskite manganites, we propose a model with two channels of electrical transport (TCET), which was derived from the IEO model. ${ }^{29,37}$ The IEO model is based on the following three postulates: (i) in a given sublattice, an $\mathrm{O} 2 \mathrm{p}$ electron with a constant spin direction can hop from an $\mathrm{O}^{2-}$ anion to an $\mathrm{O} 2 \mathrm{p}$ hole belonging to an adjacent $\mathrm{O}^{1-}$ anion, with the metal cation acting as the intermediary. (ii) The two $\mathrm{O} 2 \mathrm{p}$ electrons in the outer orbit of an $\mathrm{O}^{2-}$ anion, which have opposite spin directions, become itinerant electrons of two different sublattices (such as the $(\mathrm{A})$ and $[\mathrm{B}]$ sublattices of $(\mathrm{A})[\mathrm{B}]_{2} \mathrm{O}_{4}$ spinel ferrites, or the $\mathrm{A}$ and $\mathrm{B}$ sublattices of $\mathrm{ABO}_{3}$ manganites). (iii) Since an itinerant electron has a constant spin direction in a given sublattice and owing to Hund's rules, ${ }^{44}$ the directions of the magnetic moments of cations with $n_{\mathrm{d}} \leq 4$ (where $n_{\mathrm{d}}$ is the number of $3 \mathrm{~d}$ electrons in $3 \mathrm{~d}$ transition metal cations), such as $\mathrm{Mn}^{3+}$ or divalent and trivalent $\mathrm{Cr}$ cations, will couple antiferromagnetically with those of magnetic moments of cations with $n_{\mathrm{d}} \geq 5$, such as $\mathrm{Mn}^{2+}$ or divalent and trivalent $\mathrm{Fe}$, Co, and $\mathrm{Ni}$ cations. This occurs because in the $3 \mathrm{~d}$ orbits of transition elements, a maximum of five electrons can have their spins aligned in the same direction.

It is well known that there are two sublattices in $\mathrm{ABO}_{3}$ perovskite manganites: the $\mathrm{A}-\mathrm{O}$ sublattice and the $\mathrm{B}-\mathrm{O}$ sublattice. According to the above IEO model, there are $\mathrm{O}-\mathrm{A}-\mathrm{O}-\mathrm{A}-\mathrm{O}$ and $\mathrm{O}-\mathrm{B}-\mathrm{O}-\mathrm{B}-\mathrm{O}$ ionic chains, which can be regarded as two current-carrier channels. In $\mathrm{La}_{1-x} \mathrm{Sr}_{x} \mathrm{MnO}_{3}$, the movement of $\mathrm{O}$ $2 \mathrm{p}$ electrons along the first channel, i.e., the $\mathrm{O}-\mathrm{A}-\mathrm{O}-\mathrm{A}-\mathrm{O}$ (where La and Sr occupy the A sites) ionic chain, is a spin-independent process because there is no magnetic ordering. The resistance of the first channel is labeled $R_{3}$ (with an equivalent resistivity of $\rho_{3}$ ). The movement of $\mathrm{O} 2 \mathrm{p}$ itinerant electrons along the second channel, i.e., the O-Mn-O-Mn-O (where Mn occupies the B site in $\mathrm{O}-\mathrm{B}-\mathrm{O}-\mathrm{B}-\mathrm{O}$ ) ionic chain, is a spin-dependent process below

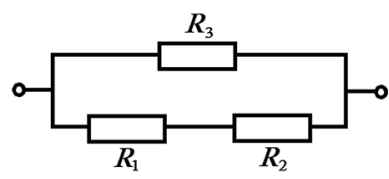

(a)

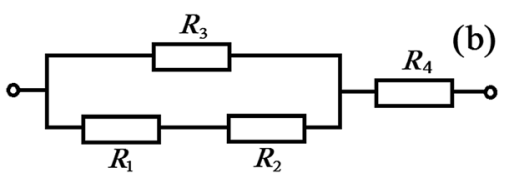

Fig. 4 The equivalent circuit of the TCET model of (a) single-crystalline and (b) polycrystalline perovskite manganites. $R_{1}$ includes the residual resistance and the resistance resulting from scattering by the crystal lattice. $R_{2}$ originates from the spin orientations of the itinerant electrons and local electrons of the outer $\mathrm{O} 2 \mathrm{p}$ and $\mathrm{Mn} 3 \mathrm{~d}$ orbitals that deviate from the orientation of their ground states because of thermal fluctuation. $R_{3}$ is spin-independent resistance. $R_{4}$ originates from the scattering by the crystallite interfaces in the polycrystalline sample.

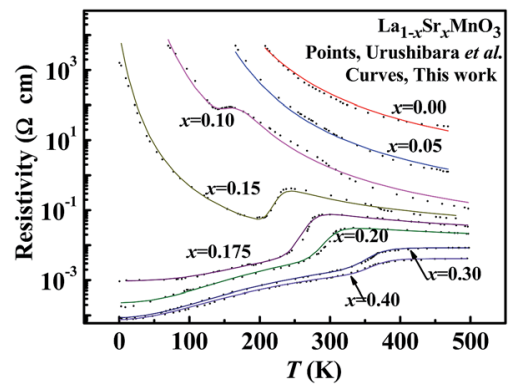

Fig. 5 Temperature dependence of electrical resistivity of the singlecrystalline $\mathrm{La}_{1-x} \mathrm{Sr}_{x} \mathrm{MnO}_{3}$ samples. The data points represent values observed by Urushibara et al., ${ }^{41}$ and the curves represent the fitted values obtained in this work.

the Curie temperature because there is magnetic ordering. The resistance of the second channel is represented by two resistors in series, $R_{1}$ and $R_{2}$ (with an equivalent resistivity of $\rho_{1}+\rho_{2}$ ), where $\rho_{1}$ includes the residual resistivity and the resistivity resulting from scattering by the crystal lattice, and $\rho_{2}$ originates from the spin orientations of the itinerant electrons and local electrons of the outer $\mathrm{O} 2 \mathrm{p}$ and $\mathrm{Mn} 3 \mathrm{~d}$ orbits that deviate from the orientation of their ground states because of thermal fluctuation. Therefore, the TCET model for a perovskite manganite sample can be represented by the equivalent circuit shown in Fig. 4(a), with the resistance, $R$, and resistivity, $\rho$, defined as follows:

$$
R=\frac{\left(R_{1}+R_{2}\right) R_{3}}{R_{1}+R_{2}+R_{3}}, \rho=\frac{\left(\rho_{1}+\rho_{2}\right) \rho_{3}}{\rho_{1}+\rho_{2}+\rho_{3}}
$$

A series of curves of $\rho$ versus test temperature, $T$, for singlecrystalline $\mathrm{La}_{1-x} \mathrm{Sr}_{x} \mathrm{MnO}_{3}(0.00 \leq x \leq 0.40)$ were reported by Urushibara et al., ${ }^{41}$ and they were fitted using the TCET model. As shown in Fig. 5, the fitted curves are very close to the observed results (data points).

When $0.175 \leq x \leq 0.40$, we found that the resistivities in eqn (1) can be expressed as follows:

$$
\begin{aligned}
& \rho_{1}=\rho_{0}+a_{1}\left(T_{1}+T\right)^{3}, \rho_{2}=a_{2} \exp \left(-\frac{E_{2}}{k_{\mathrm{B}} T}\right), \\
& \rho_{3}=a_{3} \exp \left(\frac{E_{3}}{k_{\mathrm{B}} T}\right)
\end{aligned}
$$

where $k_{\mathrm{B}}$ is the Boltzmann constant. The parameters in eqn (2) were determined by fitting the experimental curves of $\rho$ versus $T$, and the results are listed in Table 2. For example, the parameters for the sample with $x=0.20$ were determined as follows: first, it can be seen that the curve of $\rho$ versus $\left(T_{1}+T\right)^{3}$ can be approximated as a straight line at lower temperatures by adjusting the parameter $T_{1}$, as shown in Fig. 6(a). We then determined the residual resistivity, $\rho_{0}$, and the parameter $a_{1}$ from the intercept and slope, respectively, of the straight line in Fig. 6(a). Second, an approximate straight line of $\ln \rho$ versus $1 /$ $k_{\mathrm{B}} T$ can be seen at higher temperatures, as shown in Fig. 6(b). We obtained the approximate values of $\ln a_{3}$ and $E_{3}$ from the intercept and slope, respectively, of the straight line in Fig. 6(b). 
Table 2 Parameters of $\mathrm{La}_{1-x} \mathrm{Sr}_{x} \mathrm{MnO}_{3}$ obtained by fitting the experimental curves of resistivity $(\rho)$ versus test temperature ( $T$ ) reported by Urushibara et al. ${ }^{41}$ The $\rho_{0}$ is the residual resistivity in $\rho_{1}$; The $a_{1}$ and $T_{1}$ are the parameters (in the $\rho_{1}$ ) which result from scattering by the crystal lattice; The $a_{11}$ and $E_{1}$ are the parameters in the $\rho_{1}$ of the samples with $x=0.10$ and 0.15 ; The $a_{2}$ and $E_{2}$ are the parameters (in the $\rho_{2}$ ) which originate from the spin orientations of the itinerant electrons and local electrons of the outer $O 2 \mathrm{p}$ and $\mathrm{Mn} 3 \mathrm{~d}$ orbits that deviate from the orientation of their ground states because of thermal fluctuation. The $a_{3}$ and $E_{3}$ are the parameters (in the $\rho_{3}$ ) which originate from the spinindependent transition

\begin{tabular}{|c|c|c|c|c|c|c|c|c|c|}
\hline Sr content $x$ & $\rho_{0}(\Omega \mathrm{cm})$ & $a_{1}\left(\Omega \mathrm{cm} \mathrm{K}^{-3}\right)$ & $T_{1}(\mathrm{~K})$ & $a_{11}(\Omega \mathrm{cm})$ & $E_{1}(\mathrm{eV})$ & $a_{2}(\Omega \mathrm{cm})$ & $E_{2}(\mathrm{eV})$ & $a_{3}(\Omega \mathrm{cm})$ & $E_{3}(\mathrm{eV})$ \\
\hline 0.00 & - & - & - & - & - & - & - & 0.2440 & 0.175 \\
\hline 0.05 & - & - & - & - & - & - & - & 0.0220 & 0.170 \\
\hline 0.10 & - & - & - & 0.009 & 0.15 & $4 \times 10^{5}$ & 0.115 & 0.0050 & 0.150 \\
\hline 0.15 & - & - & - & 0.0014 & 0.08 & $1 \times 10^{11}$ & 0.515 & 0.0115 & 0.075 \\
\hline 0.175 & $9.5 \times 10^{-4}$ & $2.4 \times 10^{10}$ & 10 & - & - & $8 \times 10^{10}$ & 0.650 & 0.0112 & 0.0515 \\
\hline 0.20 & $2.3 \times 10^{-4}$ & $1.5 \times 10^{10}$ & 20 & - & - & $4.5 \times 10^{10}$ & 0.730 & 0.0095 & 0.0354 \\
\hline 0.30 & $8.5 \times 10^{-5}$ & $5.5 \times 10^{-11}$ & 30 & - & - & $9 \times 10^{9}$ & 0.860 & 0.0084 & $1 \times 10^{-4}$ \\
\hline 0.40 & $7.5 \times 10^{-5}$ & $4.2 \times 10^{-11}$ & 35 & - & - & $7 \times 10^{9}$ & 0.880 & 0.0041 & $5 \times 10^{-5}$ \\
\hline
\end{tabular}

Third, we determined the parameters $a_{2}$ and $E_{2}$ by fitting the curves of $\rho$ versus $T$. In this fitting process, the parameters $\rho_{0}, a_{1}$, and $T_{1}$ remained constant, but $a_{3}$ and $E_{3}$ required slight adjustments. The curves of observed $\rho$, and fitted $\rho_{1}, \rho_{2}$ and $\rho_{3}$ versus $T$ are shown in Fig. 6(c). Finally, the dependence of the observed (data points) and fitted (curve) $\rho$ of $\mathrm{La}_{0.8} \mathrm{Sr}_{0.2} \mathrm{MnO}_{3}$ on the test temperature, $T$, is shown in Fig. 6(d). It can be seen that the fitted curve is very close to the observed results.

For the antiferromagnetic semiconductor samples with $x=$ 0.00 and 0.05 , the curves of $\rho$ versus $T$ can only be fitted by the equation $\rho=a_{3} \exp \left(\frac{E_{3}}{k_{\mathrm{B}} T}\right)$. The values of the fitted parameters $a_{3}$ and $E_{3}$ are listed in Table 2.
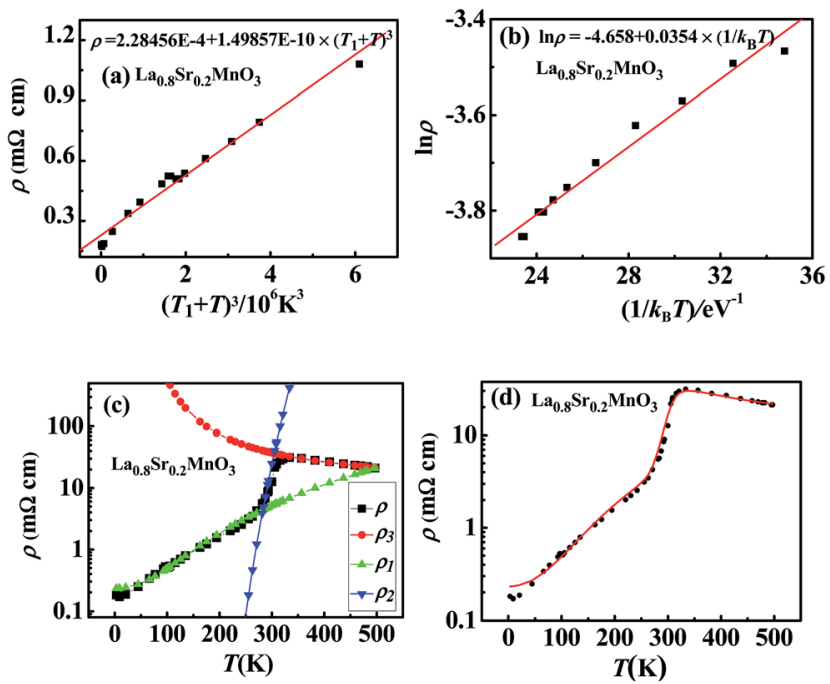

Fig. 6 Fitting process of the temperature $(T)$ dependence of the resistivity $(\rho)$ for single-crystalline $\mathrm{La}_{0.8} \mathrm{Sr}_{0.2} \mathrm{MnO}_{3}$. The data points in (a, $\mathrm{b}$ and $\mathrm{d}$ ) represent values observed by Urushibara et al. ${ }^{41}$ (a) Fitting results of the curve of $\rho$ versus $\left(T_{1}+T\right)^{3}$ at lower temperatures. (b) Fitting results of the curve of $\ln \rho$ versus $1 / k_{\mathrm{B}} T$ at higher temperatures. (c) Curves of observed $\rho$ (squares), ${ }^{41}$ fitted $\rho_{1}$ (triangles), fitted $\rho_{2}$ (inverted triangles), and fitted $\rho_{3}$ (circles) versus $T$. (d) Dependence of observed (data points ${ }^{41}$ ) and fitted (curve) $\rho$ on $T$.
For the samples with $x=0.10$ and 0.15 , the curves of $\rho$ versus $T$ can be fitted using eqn (1) and (2), but the expression $\rho_{1}=a_{11} \exp \left(\frac{E_{1}}{k_{\mathrm{B}} T}\right)$ must substitute for $\rho_{1}$ in eqn (2). The values of the fitted parameters $a_{11}, E_{1}, a_{2}, E_{2}, a_{3}$, and $E_{3}$ are listed in Table 2.

For the polycrystalline samples of $\mathrm{La}_{0.6} \mathrm{Sr}_{0.4} \mathrm{Fe}_{x} \mathrm{Mn}_{1-x} \mathrm{O}_{3}(0.00$ $\leq x \leq 0.30)$ prepared in this work, scattering by the crystallite interfaces affected the sample resistivity, $\rho$, and the equivalent circuit is shown in Fig. 4(b). We first fitted the observed curves of $\rho$ versus $T$ using eqn (1) and (2), and the effect of scattering at the crystallite interfaces was then analyzed, as discussed in following Section 4.3. The fitted curves of $\rho$ versus $T$ for $\mathrm{La}_{0.6^{-}}$ $\mathrm{Sr}_{0.4} \mathrm{Fe}_{x} \mathrm{Mn}_{1-x} \mathrm{O}_{3}(0.00 \leq x \leq 0.30)$ are shown in Fig. 3. It can be seen that the fitted curves are very close to the observed results. The values of the fitted parameters are listed in Table 3.

\section{Discussion}

4.1 Spin-dependent and spin-independent electrical transport in perovskite manganites, and their difference from free electron transport in magnetic metals

Fig. 6(c) shows that at lower temperatures, the spinindependent resistivity, $\rho_{3}$, of $\mathrm{La}_{0.8} \mathrm{Sr}_{0.2} \mathrm{MnO}_{3}$ prepared by Urushibara et al. was so high that the electrical transport occurred along the spin-dependent channel, and the value of $\rho$ was close to $\rho_{1}$, which increased with increases in the test temperature $T$ owing to the thermal vibration of ions. When $T$ was close to $T_{\mathrm{C}}$, the transition probability of the itinerant electrons decreased rapidly and $\rho_{2}$ increased rapidly because the spin orientations of the itinerant electrons and the electrons in the outer $\mathrm{O} 2 \mathrm{p}$ and $\mathrm{Mn} 3 \mathrm{~d}$ orbits deviated from the direction of their ground states. When $T$ was higher than $T_{\mathrm{C}}$, the spin orientations of the itinerant electrons changed into disorder, and the electrical transport became spin-independent along both $\mathrm{O}-\mathrm{A}-\mathrm{O}-\mathrm{A}-\mathrm{O}$ and $\mathrm{O}-\mathrm{B}-\mathrm{O}-\mathrm{B}-\mathrm{O}$ ionic chains.

It should be noted that the resistivity of $\mathrm{La}_{0.6} \mathrm{Sr}_{0.4} \mathrm{MnO}_{3}$, also prepared by Urushibara et al., was $84 \mu \Omega \mathrm{cm}$ at $2 \mathrm{~K},{ }^{41}$ which is far higher than the resistivities of the magnetic metals Fe $(8.6 \mu \Omega$ $\mathrm{cm}), \mathrm{Ni}(6.14 \mu \Omega \mathrm{cm})$, and $\mathrm{Co}(5.57 \mu \Omega \mathrm{cm})$. Therefore, it can be 
Table 3 Parameters of $\mathrm{La}_{0.6} \mathrm{Sr}_{0.4} \mathrm{Fe}_{x} \mathrm{Mn}_{1-x} \mathrm{O}_{3}$ obtained by fitting the experimental curves of $\rho$ versus $T$ from this work. The $\rho_{0}$ is the residual resistivity in $\rho_{1}$; The $a_{1}$ and $T_{1}$ are the parameters (in the $\rho_{1}$ ) which result from scattering by the crystal lattice; The $a_{2}$ and $E_{2}$ are the parameters (in the $\rho_{2}$ ) which originate from the spin orientations of the itinerant electrons and local electrons of the outer $\mathrm{O} 2 \mathrm{p}$ and $\mathrm{Mn} 3 \mathrm{~d}$ orbits that deviate from the orientation of their ground states because of thermal fluctuation. The $a_{3}$ and $E_{3}$ are the parameters (in the $\rho_{3}$ ) which originate from the spin-independent transition. All these parameters include the effect of the crystallite interfaces

\begin{tabular}{|c|c|c|c|c|c|c|c|}
\hline Fe content $x$ & $\rho_{0}(\Omega \mathrm{cm})$ & $a_{1}\left(\Omega \mathrm{cm} \mathrm{K}^{-3}\right)$ & $T_{1}(\mathrm{~K})$ & $a_{2}(\Omega \mathrm{cm})$ & $E_{2}(\mathrm{eV})$ & $a_{3}(\Omega \mathrm{cm})$ & $E_{3}(\mathrm{eV})$ \\
\hline 0 & 0.0072 & $2.794 \times 10^{-10}$ & 150 & 0.05 & 0.060 & 0.0044 & 0.0650 \\
\hline 0.05 & 0.0172 & $1.050 \times 10^{-9}$ & 107 & 0.40 & 0.090 & 0.0020 & 0.0865 \\
\hline 0.15 & 1.1000 & $1.159 \times 10^{-6}$ & 20 & 20000 & 0.120 & 0.0018 & 0.1070 \\
\hline 0.2 & - & - & - & - & - & 0.0030 & 0.1140 \\
\hline 0.25 & - & - & - & - & - & 0.0056 & 0.1200 \\
\hline
\end{tabular}

concluded that the spin-dependent transport of itinerant electrons in perovskite manganites below $T_{\mathrm{C}}$ is distinctly different from the spin-independent transport of free electrons in magnetic metals.

\subsection{Effect of proportion of the antiferromagnetic phase on the resistivity of $\mathrm{La}_{0.6} \mathrm{Sr}_{0.4} \mathrm{Fe}_{x} \mathrm{Mn}_{1-x} \mathrm{O}_{3}(0.00 \leq x \leq 0.30)$}

Our previous investigation ${ }^{37-39}$ showed that all $\mathrm{Mn}$ and $\mathrm{Fe}$ ions are trivalent in $\mathrm{La}_{0.6} \mathrm{Sr}_{0.4} \mathrm{Fe}_{x} \mathrm{Mn}_{1-x} \mathrm{O}_{3}$. According to the IEO model, an itinerant electron with up-spin in a sample with no Fe doping moves along the $\mathrm{O}^{2-}-\mathrm{Mn}^{3+}-\mathrm{O}^{1-}-\mathrm{Mn}^{3+}-\mathrm{O}^{2-}$ ion chain, which always occupies the highest energy level whenever it reaches a $\mathrm{Mn}^{3+}$ or $\mathrm{O}^{2-}$ ion, as shown in Fig. 7(a). In this transition process, the itinerant electron consumes a small fraction of the energy of the system. When an itinerant electron with upspin moves along the $\mathrm{O}^{2-}-\mathrm{Fe}^{3+}-\mathrm{O}^{1-}-\mathrm{Mn}^{3+}-\mathrm{O}^{2-}$ ion chain, it always occupies the highest energy whenever it reaches a $\mathrm{Mn}^{3+}$ or $\mathrm{O}^{1-}$ ion, but the lowest energy level of an $\mathrm{Fe}^{3+}$ ion, as shown in Fig. 7(b), because the $\mathrm{Fe}^{3+}\left(3 \mathrm{~d}^{5}\right)$ cation couples antiferromagnetically with the $\mathrm{Mn}^{3+}\left(3 \mathrm{~d}^{4}\right)$ cation (see the IEO model). In this transition process, the itinerant electron must consume more energy of the system, resulting in lower transition probabilities for the itinerant electrons and increased resistivity. Therefore, the resistivity increases with increasing proportion of the antiferromagnetic phase. As shown in Fig. 7(a and b), the

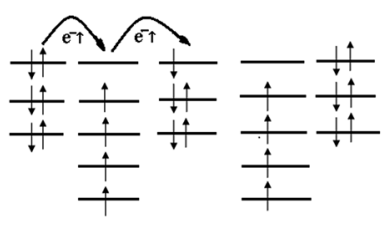

$\mathrm{O}^{2-}-\mathrm{Mn}^{3+}-\mathrm{O}^{1-}-\mathrm{Mn}^{3+}-\mathrm{O}^{2-}$

(a)

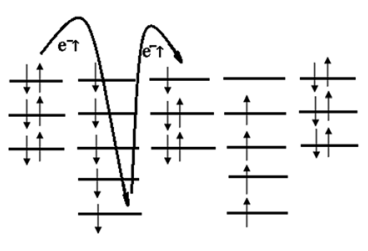

$\mathrm{O}^{2-}-\mathrm{Fe}^{3+}-\mathrm{O}^{1-}-\mathrm{Mn}^{3+}-\mathrm{O}^{2-}$

(b)
Fig. 7 Schematic diagrams of the transit of itinerant electrons in a spin-dependent process. (a) An itinerant electron with up-spin moves along the $\mathrm{O}^{2-}-\mathrm{Mn}^{3+}-\mathrm{O}^{1-}-\mathrm{Mn}^{3+}-\mathrm{O}^{2-}$ ion chain. (b) An itinerant electron moves along the $\mathrm{O}^{2-}-\mathrm{Fe}^{3+}-\mathrm{O}^{1-}-\mathrm{Mn}^{3+}-\mathrm{O}^{2-}$ ion chain. Here, the majority spin orientation of $3 d$ electrons in $\mathrm{Mn}^{3+}$ cations is up; the majority spin orientation of $3 d$ electrons in $\mathrm{Fe}^{3+}$ cations and $\mathrm{O}$ $2 \mathrm{p}$ electrons in $\mathrm{O}^{1-}$ anions is down. spin orientation of the majority of $3 \mathrm{~d}$ electrons in $\mathrm{Mn}^{3+}$ cations is up; the spin orientation of the majority of local $3 \mathrm{~d}$ electrons in $\mathrm{Fe}^{3+}$ cations and local $\mathrm{O} 2 \mathrm{p}$ electrons in $\mathrm{O}^{1-}$ anions is down.

The difference between the energy consumed by the itinerant electrons in polycrystalline $\mathrm{La}_{0.6} \mathrm{Sr}_{0.4} \mathrm{MnO}_{3}$ and $\mathrm{La}_{0.6} \mathrm{Sr}_{0.4^{-}}$ $\mathrm{Fe}_{0.1} \mathrm{Mn}_{0.9} \mathrm{O}_{3}$ was estimated from their Curie temperatures (364.5 and $267.9 \mathrm{~K}$, respectively; see Table 1 ) to be $8.3 \mathrm{meV}$.

\subsection{Resistivity $\left(\rho_{4}\right)$ originating from scattering at the crystallite interfaces of polycrystalline samples}

It is interesting to compare the resistivity of the polycrystalline $\mathrm{La}_{0.6} \mathrm{Sr}_{0.4} \mathrm{MnO}_{3}$ sample $\left(\rho_{\mathrm{P}}\right)$ prepared in this work to that of the single-crystalline $\mathrm{La}_{0.6} \mathrm{Sr}_{0.4} \mathrm{MnO}_{3}$ sample $\left(\rho_{\mathrm{S}}\right)$ reported by Urushibara et al., ${ }^{41}$ as shown in Fig. 8(a). We found that $\rho_{\mathrm{P}}$ is 97 times (at $50 \mathrm{~K}$ ) and 9 times (at $360 \mathrm{~K}$ ) the value of $\rho_{\mathrm{S}}$, which suggests that the resistivity $\left(\rho_{4}\right)$ originating from scattering at the crystallite interfaces of the polycrystalline sample was far higher those originating from lattice thermal vibrating $\left(\rho_{1}\right)$ and spin-dependent scattering $\left(\rho_{2}\right)$. Therefore, for the polycrystalline manganites, the TCET model can be represented by the equivalent circuit shown in Fig. 4(b). Fig. 8(b) shows a comparison of $\rho_{4}$ with $\rho_{\mathrm{P}}$, where $\rho_{4}$ was estimated using $\rho_{4}=\rho_{\mathrm{P}}$ $-\rho_{\mathrm{S}}$.

According to the report by Urushibara et al., ${ }^{\mathbf{4 1}}$ the temperature $T_{\mathrm{MI}}$ for maximum resistivity is very close to the value of $T_{\mathrm{C}}$ for single-crystalline $\mathrm{La}_{1-x} \mathrm{Sr}_{x} \mathrm{MnO}_{3}(0.175 \leq x \leq 0.40)$. However, as shown in Table 2 , the values of $T_{\mathrm{MI}}$ were distinctly lower than $T_{\mathrm{C}}$ for the polycrystalline $\mathrm{La}_{0.6} \mathrm{Sr}_{0.4} \mathrm{Fe}_{x} \mathrm{Mn}_{1-x} \mathrm{O}_{3}(0.00$ $\leq x \leq 0.15$ ) samples. This was simply due to $\rho_{4}$ of the polycrystalline samples. For the $\mathrm{La}_{0.6} \mathrm{Sr}_{0.4} \mathrm{MnO}_{3}$ sample, the temperature at which $\rho_{4}$ reached a maximum was $287 \mathrm{~K}$ (see Fig. 8(b)), which is distinctly lower than its $T_{\mathrm{C}}(364.5 \mathrm{~K})$.

\subsection{Effect of crystal-cell constants on the samples' resistivity}

For the antiferromagnetic semiconductor, polycrystalline $\mathrm{La}_{0.6} \mathrm{Sr}_{0.4} \mathrm{Fe}_{0.3} \mathrm{Mn}_{0.7} \mathrm{O}_{3}$, its resistivity was far lower than that of single-crystalline $\mathrm{LaMnO}_{3}$, with a $\rho_{\mathrm{P}} / \rho_{\mathrm{S}}$ ratio of $0.51 \%$ at $270 \mathrm{~K}$ and $0.58 \%$ at $300 \mathrm{~K}$, as shown in Fig. 8(c). A comparison of Fig. 8(a) and (c) shows an interesting phenomenon: the change in amplitude of the resistivity from that of the polycrystalline ferromagnetic conductor, $\mathrm{La}_{0.6} \mathrm{Sr}_{0.4} \mathrm{MnO}_{3}$, to that of the 

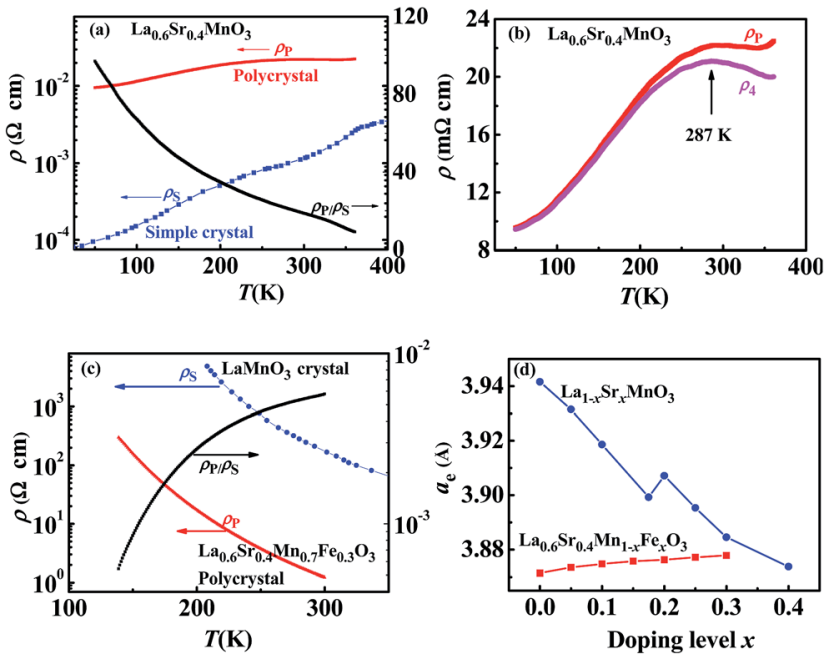

Fig. 8 (a) Temperature dependence of the resistivities, $\rho_{\mathrm{P}}$ and $\rho_{\mathrm{S}}{ }^{41}$ of polycrystalline and single-crystalline $\mathrm{La}_{0.6} \mathrm{Sr}_{0.4} \mathrm{MnO}_{3}$, and their ratio, $\rho_{\mathrm{P}} / \rho_{\mathrm{S}}$. (b) Temperature dependence of the observed resistivity, $\rho_{\mathrm{P}}$, and the estimated resistivity resulting from crystallite interface scattering, $\rho_{4}$, of polycrystalline $\mathrm{La}_{0.6} \mathrm{Sr}_{0.4} \mathrm{MnO}_{3}$, (c) Temperature dependence of $\rho_{\mathrm{P}}$ of $\mathrm{La}_{0.6} \mathrm{Sr}_{0.4} \mathrm{Fe}_{0.3} \mathrm{Mn}_{0.7} \mathrm{O}_{3}$ and $\rho_{\mathrm{S}}$ of $\mathrm{LaMnO}_{3}$, and their ratio, $\rho_{\mathrm{P}} / \rho_{\mathrm{S}}$. (d) Dependence of the equivalent cubic cell constant, $a_{\mathrm{e}}$, of the singlecrystalline $\mathrm{La}_{1-x} \mathrm{Sr}_{x} \mathrm{MnO}_{3}$ samples ${ }^{41}$ and the polycrystalline $\mathrm{La}_{0.6} \mathrm{Sr}_{0.4^{-}}$ $\mathrm{Fe}_{x} \mathrm{Mn}_{1-x} \mathrm{O}_{3}$ samples on the doping level, $x$.

antiferromagnetic semiconductor, $\mathrm{La}_{0.6} \mathrm{Sr}_{0.4} \mathrm{Fe}_{0.3} \mathrm{Mn}_{0.7} \mathrm{O}_{3}$, is far lower than the change in amplitude of the resistivity from that of the single-crystalline ferromagnetic conductor, $\mathrm{La}_{0.6} \mathrm{Sr}_{0.4^{-}}$ $\mathrm{MnO}_{3}$, to that of the antiferromagnetic semiconductor, $\mathrm{LaMnO}_{3}$. The primary causes of this phenomenon may be the distinct difference in the crystal-cell constants of the two systems, and the proportion of the antiferromagnetic phase. The equivalent cubic cell constants $\left(a_{\mathrm{e}}\right)$ of single-crystalline $\mathrm{La}_{1-x} \mathrm{Sr}_{x} \mathrm{MnO}_{3}$ (ref. 41) and polycrystalline $\mathrm{La}_{0.6} \mathrm{Sr}_{0.4} \mathrm{Fe}_{x} \mathrm{Mn}_{1-x^{-}}$ $\mathrm{O}_{3}$ are shown in Fig. 8(d), which were calculated using the average volume per formula of each sample. The samples of $\mathrm{La}_{0.6} \mathrm{Sr}_{0.4} \mathrm{Fe}_{x} \mathrm{Mn}_{1-x} \mathrm{O}_{3}(0.0 \leq x \leq 0.3)$ and $\mathrm{La}_{1-x} \mathrm{Sr}_{x} \mathrm{MnO}_{3}(0.175$ $\leq x \leq 0.40)$ exhibited rhombohedral structures, while $\mathrm{La}_{1-x^{-}}$ $\mathrm{Sr}_{x} \mathrm{MnO}_{3}(0.00 \leq x \leq 0.15)$ was reported to have an orthorhombic structure. ${ }^{41}$ The value of $a_{\mathrm{e}}$ of the polycrystalline $\mathrm{La}_{0.6} \mathrm{Sr}_{0.4} \mathrm{MnO}_{3}$ is very close to that of the single-crystalline $\mathrm{La}_{0.6} \mathrm{Sr}_{0.4} \mathrm{MnO}_{3}$. However, the value of $a_{\mathrm{e}}$ of polycrystalline $\mathrm{La}_{0.6} \mathrm{Sr}_{0.4} \mathrm{Fe}_{x} \mathrm{Mn}_{1-x} \mathrm{O}_{3}$ prepared in our study increased from $3.872 \AA(x=0.00)$ to $3.878 \AA(x=0.30)$, while the value of $a_{\mathrm{e}}$ of single-crystalline $\mathrm{La}_{1-x} \mathrm{Sr}_{x} \mathrm{MnO}_{3}$ was reported to increase from $3.874 \AA(x=0.40)$ to $3.942 \AA(x=0.00)$. According to previous investigations, ${ }^{37,38}$ there are only $\mathrm{Mn}^{3+}$ cations in $\mathrm{La}_{1-x} \mathrm{Sr}_{x} \mathrm{MnO}_{3}$ $(0.15 \leq x \leq 0.40)$. Therefore, the increase in resistivity from $x=$ 0.40 to $x=0.15$ occurred because of the increase in the crystalcell constant, while the increase in resistivity from $x=0.15$ to $x$ $=0.00$ occurred because of increases in both the crystal-cell constant and the proportion of the antiferromagnetic phase.

Obviously, an increase (in increments of $0.01 \AA$, for example) in $a_{\mathrm{e}}$ may rapidly reduce the transition probability of the itinerant electrons whenever spin-dependent or spin-independent transitions take place, resulting in an increase in the resistivity. This may be the reason the samples of $\mathrm{La}_{0.9} \mathrm{Sr}_{0.1^{-}}$ $\mathrm{MnO}_{3}$ and $\mathrm{La}_{0.85} \mathrm{Sr}_{0.15} \mathrm{MnO}_{3}$ exhibited high resistivity at low temperatures, as shown in Fig. 5. For the sample of $\mathrm{La}_{0.85^{-}}$ $\mathrm{Sr}_{0.15} \mathrm{MnO}_{3}, \rho>500 \Omega \mathrm{cm}$ when $T<10 \mathrm{~K}$ because of the large crystal lattice constant, $a_{\mathrm{e}}$, which resulted in a lower spindependent transition probability of the itinerant electrons. The value of $\rho$ decreased with increasing $T$ below $202 \mathrm{~K}$ because of the thermal energy of the itinerant electrons, which resulted in an increase in their spin-dependent transition probability. In the temperature range of $202-234 \mathrm{~K}, \rho$ increased with increasing $T$ because the spin orientation of the itinerant electrons and local electrons of the outer $\mathrm{O} 2 \mathrm{p}$ and $\mathrm{Mn} 3 \mathrm{~d}$ orbits deviated from their ground state direction, resulting in a decrease in their spin-dependent transition probability.

\subsection{Activation energy $\left(E_{3}\right)$ of spin-independent electron transition}

The values of the activation energy, $E_{3}$, of the spin-independent electron transition in the polycrystalline $\mathrm{La}_{0.6} \mathrm{Sr}_{0.4} \mathrm{Fe}_{x} \mathrm{Mn}_{1-x} \mathrm{O}_{3}$ $(0.00 \leq x \leq 0.30)$ samples ranged from 65.0 to $124.5 \mathrm{meV}$ (see Table 3), which are similar to the values (90.5 to $148.3 \mathrm{meV}$ ) of the polaron activation energy, $E_{\mathrm{p}}$, obtained by Liu et al. with the CCDC model for $\mathrm{La}_{0.7} \mathrm{Ca}_{0.3} \mathrm{Ti}_{x} \mathrm{Mn}_{1-x} \mathrm{O}_{3}(0.00 \leq x \leq 0.07) .{ }^{18}$ The comparable results indicate that the TCET model in this work is similar to the CCDC model. ${ }^{\mathbf{1 3 , 1 4 , 1 8 - 2 0}}$ However, the physical mechanism of the TCEP model, which includes spin-dependent and spin-independent electron transitions, is easier to be understood than that of the CCDC model, which assumes that the current-carrier density changes sharply at the magnetictransition temperature.

Tables 2 and 3 show that among the ferromagnetic samples, $E_{3}$ of polycrystalline $\mathrm{La}_{0.6} \mathrm{Sr}_{0.4} \mathrm{MnO}_{3}(65.0 \mathrm{meV})$ is far higher than that of single-crystalline $\mathrm{La}_{0.6} \mathrm{Sr}_{0.4} \mathrm{MnO}_{3}(0.05 \mathrm{meV})$ owing to scattering at the crystallite interfaces in the polycrystalline sample. However, among the antiferromagnetic samples, $E_{3}$ of polycrystalline $\mathrm{La}_{0.6} \mathrm{Sr}_{0.4} \mathrm{Fe}_{0.3} \mathrm{Mn}_{0.7} \mathrm{O}_{3}(124.5 \mathrm{meV})$ is distinctly lower than that of antiferromagnetic $\mathrm{LaMnO}_{3}(175 \mathrm{meV})$, which may have been caused by the different crystal-cell constants, as discussed in the previous section.

The value of the parameter $a_{3}(0.0044 \Omega \mathrm{cm}$, see Table 3) of the polycrystalline conductor, $\mathrm{La}_{0.6} \mathrm{Sr}_{0.4} \mathrm{MnO}_{3}$, is very close to that of the single-crystalline conductor, $\mathrm{La}_{0.6} \mathrm{Sr}_{0.4} \mathrm{MnO}_{3}(0.0041$ $\Omega \mathrm{cm}$, see Table 2). However, the value of $a_{3}(0.0114 \Omega \mathrm{cm})$ of the polycrystalline semiconductor, $\mathrm{La}_{0.6} \mathrm{Sr}_{0.4} \mathrm{Fe}_{0.3} \mathrm{Mn}_{0.7} \mathrm{O}_{3}$, is far lower than that of the single-crystalline semiconductor, $\mathrm{LaMnO}_{3}(0.2440 \Omega \mathrm{cm})$. This may be attributed to the effect of the crystal-cell constant, as discussed in Section 4.4.

\subsection{Regression coefficients of $\rho_{1}$ and $\rho_{2}$}

As shown in eqn (2) and Tables 2 and 3, the spin-dependent resistivities, $\rho_{1}$ and $\rho_{2}$, are represented by parameters (or regression coefficients) that play interesting roles:

(i) The resistivity $\rho_{1}$ in eqn (2) is determined by three parameters: the residual resistivity, $\rho_{0}$; the parameters $a_{1}$ and $T_{1}$ resulting from scattering by the crystal lattice. All three parameters of the polycrystalline $\mathrm{La}_{0.6} \mathrm{Sr}_{0.4} \mathrm{Fe}_{x} \mathrm{Mn}_{1-x} \mathrm{O}_{3}$ 
samples $-\rho_{0}=0.0072$ to $1.100 \Omega \mathrm{cm}, a_{1}=2.794 \times 10^{-10}$ to $1.159 \times 10^{-6}(\Omega \mathrm{cm}) \mathrm{K}^{-3}, T_{1}=150$ to $20 \mathrm{~K}$-are higher than those of the single-crystalline $\mathrm{La}_{1-x} \mathrm{Sr}_{x} \mathrm{MnO}_{3}$ samples- $\rho_{0}=$ 0.075 to $0.95 \mathrm{~m} \Omega \mathrm{cm}, a_{1}=4.2 \times 10^{-11}$ to $2.4 \times 10^{-10}(\Omega \mathrm{cm})$ $\mathrm{K}^{-3}$, and $T_{1}=35$ to $10 \mathrm{~K}$-because of the effect of crystallite interfaces in the polycrystalline samples.

(ii) The resistivity $\rho_{2}$ in eqn (2) is determined by two parameters: the amplitude $a_{2}$; the activation energy $E_{2}$, which originated from spin-dependent scattering. The value of $E_{2}$ of the single-crystalline $\mathrm{La}_{1-x} \mathrm{Sr}_{x} \mathrm{MnO}_{3}$ samples increased from $0.515 \mathrm{eV}(x=0.15)$ to $0.88 \mathrm{eV}(x=0.40)$, which was the result of competition between two factors. One factor was the increase in the cant angle between magnetic moments of $\mathrm{Mn}^{3+}$ cations from $0(x=0.15)$ to $34.9^{\circ}(x=0.40) . .^{37}$ Another factor was the decrease in crystal cell volume with increasing $x$. The value of $E_{2}$ of the polycrystalline $\mathrm{La}_{0.6} \mathrm{Sr}_{0.4} \mathrm{Fe}_{x} \mathrm{Mn}_{1-x} \mathrm{O}_{3}$ samples increased from $0.06 \mathrm{eV}(x=0.00)$ to $0.12 \mathrm{eV}(x=0.15)$, which can be attributed to the increase in Fe-doping level because the magnetic moments of $\mathrm{Fe}^{3+}$ cations couple antiferromagnetically with those of $\mathrm{Mn}^{3+}$ cations. ${ }^{39}$ The $a_{2}$ values of the polycrystalline $\mathrm{La}_{0.6} \mathrm{Sr}_{0.4} \mathrm{Fe}_{x} \mathrm{Mn}_{1-x} \mathrm{O}_{3}$ samples are far lower than those of the single-crystalline $\mathrm{La}_{1-x} \mathrm{Sr}_{x} \mathrm{MnO}_{3}$ samples, possibly because of the intense scattering at the crystallite interfaces to reduce the effect of spin-dependent scattering.

\section{Conclusion}

In order to explain the dependence of the resistivity $(\rho)$ of perovskite manganites on the test temperature $(T)$, we propose a model with two channels of electrical transport (TCET): a spin-independent channel (with resistivity $\rho_{3}$ ) and a spin-dependent channel (with resistivity $\rho_{1}+\rho_{2}$ ). At low temperature, $\rho_{3}$ is very high, and the electrical transport occurs along the spin-dependent channel. When $T$ is far lower than the Curie temperature $\left(T_{\mathrm{C}}\right), \rho$ is very close to $\rho_{1}$, which increases with increasing $T$ owing to the thermal vibration of the crystal lattice. When $T$ is close to $T_{\mathrm{C}}, \rho_{2}$ increases rapidly owing to the spin orientations of the itinerant electrons, and the local electrons of the outer $\mathrm{O} 2 \mathrm{p}$ and $\mathrm{Mn} 3 \mathrm{~d}$ orbits deviating from the orientation of their ground states. Therefore, the spin-dependent electrical transport in perovskite manganites at temperatures below $T_{\mathrm{C}}$ is distinctly different from the spin-independent electrical transport in metals. When $T$ is higher than $T_{\mathrm{C}}$, the spin orientations of the itinerant electrons change into disorder, and electrical transport occurs along both channels, with the spin-independent transition, $\rho$, being close to $\rho_{3}$.

Several determining factors of the resistivity have been discussed: (i) the increase in the proportion of the antiferromagnetic phase may result in an increase in $\rho$. (ii) The resistivity $\rho_{4}$ originating from crystallite-interface scattering in the polycrystalline samples is far higher than $\rho_{1}$ and $\rho_{2}$ at temperatures below $T_{\mathrm{C}}$. (iii) Increases in the crystal-cell constant, in increments as small as $0.01 \AA$, may result in rapid increases in $\rho$, which may be the reason of the high resistivity of $\mathrm{La}_{0.9} \mathrm{Sr}_{0.1^{-}}$ $\mathrm{MnO}_{3}$ and $\mathrm{La}_{0.85} \mathrm{Sr}_{0.15} \mathrm{MnO}_{3}$ at low temperatures.

\section{Conflicts of interest}

There are no conflicts to declare.

\section{Acknowledgements}

This work is supported by the National Natural Science Foundation of China (NSF-11174069), the Natural Science Foundation of Hebei Province (A2015205111), the Key item Science Foundation of Hebei Province (Grant No. 16961106D), and the Young scholar Science Foundation of the Education Department of Hebei Province (QN2016015).

\section{References}

1 Y. Izuchi, M. Akaki, D. Akahoshi and H. Kuwahara, APL Mater., 2014, 2, 022106.

2 K. Horiba, M. Kitamura, K. Yoshimatsu, M. Minohara, E. Sakai, M. Kobayashi, A. Fujimori and H. Kumigashira, Phys. Rev. Lett., 2016, 116, 076401.

3 A. Belkahla, K. Cherif, J. Dhahri, K. Taibi and E. K. Hlil, RSC Adv., 2017, 7, 30707.

4 A. O. Turky, M. M. Rashad, A. M. Hassan, E. M. Elnaggar and M. Bechelany, RSC Adv., 2016, 6, 17980.

5 J. Lu, Y. H. Zhang, Z. Lu, X. Q. Huang, Z. H. Wang, X. B. Zhu and B. Wei, RSC Adv., 2015, 5, 5858.

6 R. Nath and A. K. Raychaudhuri, RSC Adv., 2015, 5, 57875.

7 A. Demont and S. Abanades, RSC Adv., 2014, 4, 54885.

8 A. Herpers, K. J. O'Shea, D. A. MacLaren, M. Noyong, B. Rösgen, U. Simon and R. Dittmann, APL Mater., 2014, 2, 106106.

9 C. Adamo, L. Méchin, T. Heeg, M. Katz, S. Mercone, B. Guillet, S. Wu, J.-M. Routoure, J. Schubert, W. Zander, R. Misra, P. Schiffer, X. Q. Pan and D. G. Schlom, APL Mater., 2015, 3, 062504.

10 D. J. Singh and W. E. Pickett, Phys. Rev. B: Condens. Matter Mater. Phys., 1998, 57, 88-91.

11 J. D. Lee and B. I. Min, Phys. Rev. B: Condens. Matter Mater. Phys., 1997, 55, 12454-12459.

12 H. Y. Hwang, S.-W. Cheong, N. P. Ong and B. Batlogg, Phys. Rev. Lett., 1996, 77, 2041-2044.

13 A. S. Alexandrov and A. M. Bratkovsky, Phys. Rev. Lett., 1999, 82, 141-144.

14 A. S. Alexandrov, A. M. Bratkovsky and V. V. Kabanov, Phys. Rev. Lett., 2006, 96, 117003.

15 N. Nücker, J. Fink, J. C. Fuggle, P. J. Durham and W. M. Temmerman, Phys. Rev. B: Condens. Matter Mater. Phys., 1988, 37, 5158-5163.

16 H. L. Ju, H.-C. Sohn and K. M. Krishnan, Phys. Rev. Lett., 1997, 79, 3230-3233.

17 K. Ibrahim, H. J. Qian, X. Wu, M. I. Abbas, J. O. Wang, C. H. Hong, R. Su, J. Zhong, Y. H. Dong, Z. Y. Wu, L. Wei, D. C. Xian, Y. X. Li, G. J. Lapeyre, N. Mannella, C. S. Fadley and Y. Baba, Phys. Rev. B: Condens. Matter Mater. Phys., 2004, 70, 224433.

18 X. M. Liu, H. Zhu and Y. H. Zhang, Phys. Rev. B: Condens. Matter Mater. Phys., 2001, 65, 024412. 
19 L. M. Wang, C. Y. Wang and C. C. Tseng, Appl. Phys. Lett., 2012, 100, 232403.

20 M. A. B. Narreto, H. S. Alagoz, J. Jeon, K. H. Chow and J. Jung, J. Appl. Phys., 2014, 115, 223905.

21 L. S. Xu, J. Y. Fan, Y. Zhu, Y. G. Shi, L. Zhang, L. Pi, Y. H. Zhang and D. N. Shi, Chem. Phys. Lett., 2015, 634, 174-178.

22 R. E. Cohen, Nature, 1992, 358, 136.

23 R. E. Cohen and H. Krakauer, Phys. Rev. B: Condens. Matter Mater. Phys., 1990, 42, 6416.

24 J.-C. Dupin, D. Gonbeau, P. Vinatier and A. Levasseur, Phys. Chem. Chem. Phys., 2000, 2, 1319.

25 L. Q. Wu, Y. C. Li, S. Q. Li, Z. Z. Li, G. D. Tang, W. H. Qi, L. C. Xue, X. S. Ge and L. L. Ding, AIP Adv., 2015, 5, 097210.

26 L. Q. Wu, S. Q. Li, Y. C. Li, Z. Z. Li, G. D. Tang, W. H. Qi, L. C. Xue, L. L. Ding and X. S. Ge, Appl. Phys. Lett., 2016, 108, 021905.

27 D. H. Ji, G. D. Tang, Z. Z. Li, X. Hou, Q. J. Han, W. H. Qi, R. R. Bian and S. R. Liu, J. Magn. Magn. Mater., 2013, 326, 197-200.

28 G. D. Tang, Q. J. Han, J. Xu, D. H. Ji, W. H. Qi, Z. Z. Li, Z. F. Shang and X. Y. Zhang, Phys. B, 2014, 438, 91-96.

29 J. Xu, L. Ma, Z. Z. Li, L. L. Lang, W. H. Qi, G. D. Tang, L. Q. Wu, L. C. Xue and G. H. Wu, Phys. Status Solidi B, 2015, 252, 2820-2829.

30 Z. F. Shang, W. H. Qi, D. H. Ji, J. Xu, G. D. Tang, X. Y. Zhang, Z. Z. Li and L. L. Lang, Chin. Phys. B, 2014, 23, 107503.

31 L. L. Lang, J. Xu, W. H. Qi, Z. Z. Li, G. D. Tang, Z. F. Shang, X. Y. Zhang, L. Q. Wu and L. C. Xue, J. Appl. Phys., 2014, 116, 123901.
32 X. Y. Zhang, J. Xu, Z. Z. Li, W. H. Qi, G. D. Tang, Z. F. Shang, D. H. Ji and L. L. Lang, Phys. B, 2014, 446, 92-99.

33 L. C. Xue, L. L. Lang, J. Xu, Z. Z. Li, W. H. Qi, G. D. Tang and L. Q. Wu, $A I P A d v .$, 2015, 5, 097167.

34 L. L. Lang, J. Xu, Z. Z. Li, W. H. Qi, G. D. Tang, Z. F. Shang, X. Y. Zhang, L. Q. Wu and L. C. Xue, Phys. B, 2015, 462, 4753.

35 J. Xu, D. H. Ji, Z. Z. Li, W. H. Qi, G. D. Tang, X. Y. Zhang, Z. F. Shang and L. L. Lang, Phys. Status Solidi B, 2015, 252, 411-420.

36 J. Xu, W. H. Qi, D. H. Ji, Z. Z. Li, G. D. Tang, X. Y. Zhang, Z. F. Shang and L. L. Lang, Acta Phys. Sin., 2015, 64, 017501.

37 L. Q. Wu, W. H. Qi, X. S. Ge, D. H. Ji, Z. Z. Li, G. D. Tang and W. Zhong, Europhys. Lett., 2017, 120, 27001.

38 X. S. Ge, L. Q. Wu, S. Q. Li, Z. Z. Li, G. D. Tang, W. H. Qi, H. J. Zhou, L. C. Xue and L. L. Ding, AIP Adv., 2017, 7, 045302. 39 X. S. Ge, Z. Z. Li, W. H. Qi, D. H. Ji, G. D. Tang, L. L. Ding, J. J. Qian and Y. N. Du, AIP Adv., 2017, 7, 125002.

40 L. Q. Wu, W. H. Qi, Y. C. Li, S. Q. Li, Z. Z. Li, G. D. Tang, L. C. Xue, X. S. Ge and L. L. Ding, Acta Phys. Sin., 2016, 65, 027501.

41 A. Urushibara, Y. Moritomo, T. Arima, A. Asamitsu, G. Kido and Y. Tokura, Phys. Rev. B: Condens. Matter Mater. Phys., 1995, 51, 14103.

42 D. H. Ji, G. D. Tang, Z. Z. Li, Q. J. Han, X. Hou, R. R. Bian and S. R. Liu, J. Appl. Phys., 2012, 111, 113902.

43 X. Hou, D. H. Ji, W. H. Qi, G. D. Tang and Z. Z. Li, Chin. Phys. $B, 2015,24,057501$.

44 C. W. Chen, Magnetism and Metallurgy of Soft Magnetic Materials, North-Holland Publishing Company, 1977. 